\title{
A GENERALIZATION OF SINGER'S THEOREM
}

\author{
MARK P. HALE, JR. AND DIETER JUNGNICKEL ${ }^{1}$
}

\begin{abstract}
Finite pappian Klingenberg planes and finite desarguesian uniform Hjelmslev planes admit abelian collineation groups acting regularly on the point and line sets; this generalizes Singer's theorem on finite desarguesian projective planes.
\end{abstract}

Introduction. Projective Klingenberg and Hjelmslev planes (briefly $K$ planes, resp., $H$-planes) are generalizations of ordinary projective planes. The more general $K$-plane is an incidence structure $\Pi$ whose point and line sets are partitioned into "neighbor classes" such that nonneighbor points (lines) have exactly one line (point) in common and such that the "gross structure" formed by the neighbor classes is an ordinary projective plane $\Pi^{\prime}$. An $H$-plane is a $K$-plane whose points are neighbors if and only if they are joined by at least two lines, and dually. A finite $K$-plane possesses two integer invariants $(t, r)$, where $r$ is the order of $\Pi^{\prime}$ and $t^{2}$ the cardinality of any neighbor class of $\Pi$ (cf. [3], [6]).

Examples of $K$-planes are the "desarguesian" $K$-planes which are constructed by using homogeneous coordinates over a local ring (similar to the construction of the desarguesian projective planes from skewfields); for the precise definition, see Lemma 2 . If $R$ is a finite local ring with maximal ideal $M$, one obtains a $(t, r)-K$-plane with $t=|M|$ and $r=|R / M|$. The $K$-plane will be an $H$-plane if and only if $R$ is a Hjelmslev ring (see [7] and [8]).

We recall that a projective plane is called cyclic if it admits a cyclic collineation group which acts regularly on the point and line sets. Singer's theorem [10] asserts that every finite desarguesian projective plane is cyclic. The notion of a cyclic projective plane was generalized by Jungnickel to that of a "regular" $K$-plane: the main requirement is the existence of an abelian collineation group acting regularly on the point and line sets (see Definition 2). In [4], a particular class of commutative $H$-rings was shown to give rise to regular $H$-planes by a computational argument. In the present paper, we prove a generalization of Singer's theorem: Any finite pappian $K$-plane is

Received by the editors June 1, 1977.

AMS (MOS) subject classifications (1970). Primary 50D35, 05B25; Secondary 05B 10.

Key words and phrases. Hjelmslev plane, Klingenberg plane, Singer's theorem, regular collineation group.

'The second author acknowledges the hospitality of the University of Florida during the time of his research. 
regular. As regarding nonpappian planes, we show thay any finite desarguesian $H$-plane with $t=r$ is regular.

We will only consider finite incidence structures. All rings considered are associative with unit.

The following lemma is well known (see e.g. [1, Chapitre 2]).

LEMMA 1. In a local ring $R$, the (unique) maximal ideal $M$ consists precisely of the nonunits of $R$. If $R$ is finite, $M$ is nilideal.

Definition 1 (KLingenberg). A local ring $R$ is called a Hjelmslev ring if the maximal ideal $M$ consists precisely of the zero divisors of $R$ and if for all $a, b \in M$

(i) $a \in b M$ or $b \in a M$ and

(ii) $a \in M b$ or $b \in M a$.

LEMMA 2 (KLINGENBERG). Let $R$ be a finite local ring with maximal ideal $M$, $R^{*}=R \backslash M$ be the set of units of $R$. Define an incidence structure $\Pi(R)$ as follows:

points are the homogeneous triples $R^{*} \mathbf{p}=R^{*}\left(p_{0}, p_{1}, p_{2}\right)$ where not all $p_{i}$ are in $M$;

lines are the homogeneous triples $\mathbf{u}^{T} R^{*}=\left(u_{0}, u_{1}, u_{2}\right)^{T} R^{*}$ where not all $u_{i}$ are in $M$;

incidence is defined by $R^{*} \mathbf{p} I \mathbf{u}^{T} R^{*}$ if and only if $\mathbf{p u}^{T}=0$.

Then $\Pi(R)$ is a $(t, r)$-K-plane with $t=|M|$ and $r=|R / M| . \Pi(R)$ is an $H$-plane if and only if $R$ is a Hjelmslev ring. Any nonsingular $3 \times 3$-matrix $A$ over $R$ induces a collineation of $\Pi(R)$ by putting

$$
R^{*} \mathrm{p} \mapsto R^{*}\left(\mathbf{p} A^{-1}\right) \text { and } \mathbf{u}^{T} R^{*} \mapsto\left(\mathrm{Au}^{T}\right) R^{*} .
$$

For a proof, see [7] and [8].

Definition 2. A $K$-plane $\Pi$ is called desarguesian if it can be represented as a $K$-plane $\Pi(R)$ over a local ring $R$, as in Lemma 2 . If $R$ is commutative, $\Pi$ is called pappian.

Definition 3 (JungNickel [4]). A $K$-plane $\Pi$ is called regular if it admits an abelian collineation group $G=Z \oplus K$ satisfying

(i) $G$ acts regularly on the point and line sets of $\Pi$;

(ii) $K$ acts regularly on each neighbor class (of points or lines) of $\Pi$.

One notes that (ii) is equivalent to

(ii') $Z$ acts regularly on the point and line sets of $\Pi^{\prime}$.

PROPOSITION. Let $R$ be a finite commutative local ring with maximal ideal $M$. Then there is a ring structure definable on $S=R \oplus R \oplus R$ in which $S$ is a commutative local ring extending $R$ and having maximal ideal $N=M \oplus M \oplus$ $M$.

Proof. $R / M$ is a finite field $F$. Choose a monic cubic polynomial $\bar{f}(x)$ over $F$, and let $f(x)$ be any monic cubic preimage of $\bar{f}(x)$ with respect to the natural map $R[x] \rightarrow F[x]$. We will show that $S:=R[x] /(f)$ has the desired 
properties. Certainly $S$ is a commutative extension of $R$, which appears in $S$ as the image of the constant polynomials. As an $R$-module, $S=R \oplus R \oplus R$ since $f$ is cubic. Now consider the ideal $N=(M)=S M$. Then

$$
\begin{aligned}
S / N & =R[x] /(f) /(M[x]+(f)) /(f) \cong R[x] /(M[x]+(f)) \\
& \cong R[x] / M[x] /(M[x]+(f)) / M[x]=F[x] /(\bar{f}) .
\end{aligned}
$$

By the construction of $f$, this last quotient is a 3-dimensional field extension $\bar{F}$ of $F$. Thus $N$ is maximal in $S$. Since $M$ is a nilideal in $R$, and $S$ is commutative, the ideal $N=S M$ is also a nilideal and therefore lies in every maximal ideal of $S$. This shows that $N$ is the unique maximal ideal of $S$, and $S$ is local.

THEOREM 1. A finite pappian $K$-plane $\Pi$ is regular.

Proof. Let $\Pi$ be coordinatized over the commutative local ring $R$, say $|R|=q^{n+1},|N|=q^{n}, M$ the maximal ideal of $R$. (So $\Pi$ is a $\left(q^{n}, q\right)-K$-plane.) Let $S$ be a 3-dimensional extension of $R$, as constructed in the Proposition.

Let $a \in S^{*}:=S \backslash N$ (the group of units of $S$ ). Then the mapping $\phi_{a}$ : $R^{3} \rightarrow R^{3}$ (i.e., $\phi_{a}: S \rightarrow S$ ) with $\phi_{a}: x \mapsto x a$ is a bijective linear mapping of $R^{3}$. Hence $S^{*}$ is a group of linear transformations of $R^{3}$.

But $\Pi$ is coordinatized by homogeneous triples $R^{*}\left(p_{0}, p_{1}, p_{2}\right)$, resp., $\left(u_{0}, u_{1}, u_{2}\right)^{T} R^{*}$ of elements of $R$, i.e., $\Pi$ is coordinatized by the elements of $S^{*} / R^{*}$. Thus $G:=S^{*} / R^{*}$ acts as a transitive abelian collineation group of $\Pi$. For reasons of cardinality, $G$ must be regular on $\Pi$ : $G$ has $\left(q^{3 n+3}-\right.$ $\left.q^{3 n}\right) /\left(q^{n+1}-q^{n}\right)=q^{2 n}\left(q^{2}+q+1\right)$ elements, which agrees with the number of points (resp. lines) of $\Pi$ (cf. [3] and [6]).

As $G$ is abelian and $\left(q^{2 n}, q^{2}+q+1\right)=1, G$ splits into $G=Z \oplus K$, where $Z$ has order $q^{2}+q+1$ and $K$ has order $q^{2 n}$. Consider the homomorphism from $S$ to $\bar{F}$. This induces a homomorphism from $\Pi$ (as coordinatized by $S^{*} / R^{*}$ ) to $\Pi^{\prime}$ (as coordinatized by $\bar{F}^{*} / F^{*}$ ). The image of $S^{*}$ induces a Singer cycle in $\Pi^{\prime}$, i.e., a cyclic group of order $q^{2}+q+1$; by reasons of cardinality, this is the image of $Z$; so $\Pi$ is regular with respect to $G$.

Theorem 1 yields together with [4, Corollary 2.8] a more elegant proof of the following result, which is crucial for the recursive construction of regular $H$-planes (cf. [4, §5] and [5]).

Corollary (Jungnickel [4, Corollary 4.3]). There exist $\left(q^{n}, q\right)$ - $H$-matrices for all prime powers $q$ and all natural numbers $n$.

Definition 3 (LüneBURG [9]). A $(t, r)-H$-plane $\Pi$ is called uniform if $t=r$.

THEOREM 2. A finite uniform desarguesian H-plane $\Pi$ is regular.

Proof. $\Pi$ is represented over a "uniform" Hjelmslev ring $R$ (i.e., $M^{2}=0$; see [2]). If $R$ is in fact commutative, the assertion follows by Theorem 1 . Otherwise, Theorem 6 of [2] asserts that $R$ has the following structure: Let 
$F \equiv \mathrm{GF}(q):=R / M . R$ is defined on $F \times F$ by componentwise addition and multiplication $(a, b)(c, d):=\left(a c, a d+b c^{\sigma}\right)$, where $\sigma$ is a nontrivial automorphism of $\boldsymbol{F}$. (These $\boldsymbol{H}$-rings are due to Kleinfeld [6].)

Consider the polynomial $p(x):=x^{3}-x$ over $F, p$ is not one-to-one (as $p(0)=p(1)=0)$, hence not onto; let $u$ be chosen such that $x^{3}-x-u$ has no root and is therefore irreducible. This defines, by adjoining a root $\alpha$, the 3-dimensional extension field $\operatorname{GF}\left(q^{3}\right)$. Multiplication by $\alpha(x \mapsto x \alpha)$ induces a linear transformation on $F^{3}$; its matrix is

$$
S=\left(\begin{array}{lll}
0 & 0 & 1 \\
1 & 0 & u \\
0 & 1 & 0
\end{array}\right)
$$

As in the proof of Singer's theorem one shows that $S$ induces a regular collineation group $Z$ on the projective plane $\Pi^{\prime}$ over $F$; as $F$ is a subring of $R$, it is easy to see that $Z$ in fact acts semiregularly on $\Pi$.

For $a, b \in M$, define a matrix $M(a, b)$ by

$$
M(a, b):=\left(\begin{array}{ccc}
1 & b & a \\
a & 1+u b & b+u a \\
b & a & 1+u b
\end{array}\right)
$$

(This choice of $M$ follows the ideas in the proof of Theorem 4.1 of [4].) It is easily checked that the matrices $M(a, b)$ form an abelian group isomorphic to $E A\left(q^{2}\right)$ (in fact, $M(a, b) M\left(a^{\prime}, b^{\prime}\right)=M\left(a+a^{\prime}, b+b^{\prime}\right)$, where one uses $M^{2}$ $=0)$.

Define a bijective map $\tau$ on $R$ by $(a, b) \mapsto\left(a^{\sigma^{-1}}, b^{\sigma^{-1}}\right)$. One checks that $\tau$ is an automorphism of $R$ and that $\operatorname{SM}(a, b)=M(a, b) S^{\tau}$. We remark that e.g. the $(1,3)$-entries have the form $1+u b$ and $1+b u^{\tau}$, respectively. Returning to explicit notation, i.e. writing $(u, 0)$ for $u$ and $(0, b)$ for $b$, we obtain $u b=(u, 0)(0, b)=(0, u b)$ and $b u^{\tau}=(0, b)(u, 0)^{\tau}=(0, b)\left(u^{\sigma^{-1}}, 0\right)=$ $\left(0, b\left(u^{\sigma^{-1}}\right) \sigma\right)=(0, b u)$, i.e. the desired equality. Similar computations apply for the (2, 3)- and $(3,3)$-entries. Hence, for all $\mathbf{x} \in R^{3}$,

$$
S M(a, b)\left(\mathbf{x}^{T}\right)^{\tau}=M(a, b) S^{\tau}\left(\mathbf{x}^{T}\right)^{\tau}=M(a, b)\left(\mathbf{S x}^{T}\right)^{\tau} .
$$

Hence, if we denote by $\phi(a, b)$ the collineation induced by

$$
R^{*} \mathbf{p} \mapsto R^{*} \mathbf{p}^{\tau} M(a, b)^{-1}, \quad \mathbf{u}^{T} R^{*} \mapsto M(a, b)\left(\mathbf{u}^{T}\right)^{\tau} R^{*}
$$

and by $\phi$ the collineation induced by $S$, we obtain

$$
\phi \circ \phi(a, b)=\phi(a, b) \circ \phi \text { for all } a, b \in M \text {. }
$$

Thus the abelian groups $K$ of all $\phi(a, b)$ and $Z=\langle\phi\rangle$ commute elementwise; also clearly $Z \cap K=1$, thus $G=Z \oplus K$.

It is easily checked, that $K$ is regular on the neighbor classes of the point $R^{*}(1,0,0)$ and the line $(1,0,0)^{T} R^{*}$. Thus, as $Z$ is regular on the points and lines of $\Pi^{\prime}$ (i.e., the sets of neighbor classes of points, resp., lines), $G$ is regular on the point and lines of $\Pi$ and $K$ is regular on each neighbor class of $\Pi$. 


\section{REFERENCES}

1. N. Bourbaki, Éléments de mathématique. Fasc. XXVII. Algèbre commutative, Hermann, Paris, 1961.

2. A. Cronheim, Dual numbers, Witt vectors, and Hjelmslev planes (to appear).

3. D. A. Drake and H. Lenz, Finite Klingenberg planes, Abh. Math. Sem. Hamburg 44 (1975), $70-83$.

4. D. Jungnickel, Regular Hjelmslev planes, J. Combinatorial Theory Ser. A (to appear).

5. __ Regular Hjelmslev planes. II, Trans. Amer. Math. Soc. (to appear).

6. E. Kleinfeld, Finite Hjelmslev planes, Illinois J. Math. 3 (1959), 403-407.

7. W. Klingenberg, Projektive und affine Ebenen mit Nachbarelementen, Math. Z. 60 (1954), 384-406.

8. __ Projektive Geometrien mit Homomorphismus, Math. Ann. 132 (1956), 180-200.

9. H. Lüneburg, Affine Hjelmslev-Ebenen mit transitiver Translationsgruppe, Math. Z. 79 (1962), 260-288.

10. J. Singer, $A$ theorem in finite projective geometry and some applications to number theory, Trans. Amer. Math. Soc. 43 (1938), 377-385.

Department of Mathematics, University of Florida, GainesVille, Florida 32611

Mathematisches Institut der Freien Universttät, KÖNigin-LuISSE-STRASSE 24/26, D-1000 Berlin 33, Federal Republic of Germany 